\title{
Effect of community-based nutrition on infant nutrition and associated health practices in Narok, Kenya
}

\author{
Job Mapesa, Joyce Meme, Olive Muthamia
}

Kenya Methodist University Nairobi Campus, Public Health Human Nutrition and Dietetics.

\begin{abstract}
Background: Lack of knowledge, and poor attitudes and practices among rural women have been shown to negatively influence maternal, infant and young child nutrition outcomes as well as child health and cognitive development.

Objectives: The aim of the study was to assess the impact of community-based nutrition on infant nutrition.

Methods: A mixed method approach using a structured questionnaire to collect quantitative data ( $\mathrm{n}=234)$ and Focus Group Discussions (FGDs) for qualitative data was used in Narok County, Kenya.

Results: About $57 \%$ of the participants received nutrition and health information from hospital trained health personnel. Whereas most women indicated having attended antenatal clinics, very few delivered in the hospital (17\%). Exclusive breastfeeding knowledge was below $50 \%$, although not statistically significant $(\mathrm{p}=0.584)$ across the education spectrum as opposed to identification of malnutrition signs, which was significant $(\mathrm{p}<0.05)$. Whereas $74 \%$ of the participants had knowledge about signs of malnutrition, only $58 \%$ could identify the causes. Rating for complementary feeding among the study participants was about $61 \%$ for introduction of complementary foods and $80 \%$ for frequency of feeding. Vitamin A supplementation knowledge, antenatal clinic attendance and type of waste disposal were statistically significant $(p<0.05)$ in relation to education level of the study participants.

Conclusion: This study reveals the need to educate rural women for increased understanding and practice of appropriate infant and nutrition care through sustainable and effective essential nutrition actions.

Keywords: Community; nutrition; breastfeeding; complementary foods; health; practice.

DOI: https://doi.org/10.4314/ahs.v20i2.24

Cite as: Mapesa J, Meme J, Muthamia O. Effect of community-based nutrition on infant nutrition and associated health practices in Narok, Kenya. Afri Health Sci. 2020; 20(2): 724-734. https:// doi.org/10.4314/abs.v20i2.24
\end{abstract}

\section{Introduction}

Infant and young child feeding (IYCF) practices directly affect the nutritional status of children under 2-years of age and, ultimately, impact child survival. Breastfeeding recommendations include initiation of breastfeeding within one hour of birth, exclusive breastfeeding for 6-months and continued breastfeeding for at least 2 -years with the introduction of appropriate complementary foods ${ }^{1}$. Promoting these feeding practices in children between the ages of $0-23$ months is therefore

\section{Corresponding author: \\ Job Mapesa, \\ Kenya Methodist University Nairobi Campus, \\ Public Health Human Nutrition and Dietetics. \\ Email: job.mapesa@kemu.ac.ke, \\ jmapesa@yahoo.com}

an important action towards creating an enabling environment for improved nutrition, given that $90 \%$ of the global burden of under-nutrition exists in the developing world ${ }^{2}$. To prevent malnutrition, infants must be exclusively breastfed in the first 6-months after birth ${ }^{3}$. Research shows that malnutrition can be prevented through proven nutrition intervention programs such as optimal breastfeeding and complementary feeding practices ${ }^{4,5}$. Lack of knowledge, and poor attitude and practices among rural women have been shown to negatively influence maternal, infant and young child nutrition outcomes ${ }^{6}$ and many of the nutrition intervention programs and behavior change interventions are usually implemented without community participation, a fact that greatly hampers the long-term public health outcomes $^{7}$. The benefits of a participatory approach include; development of shared visions, collective ideas, perceptions, and pooled resources that improve program outcomes and sustainability. 
In the absence of proper nutrition, children are susceptible to frequent and severe childhood infections including diarrhea, delayed cognitive development, and stunting ${ }^{8,9}$. To prevent such incidences, community-based nutrition support in vulnerable rural communities (i.e. Suswa in Narok County, Kenya) is mostly carried out by non-governmental organizations. In the last decade there has been a substantial increase in donor funding to support non-governmental organizations running such nutrition programs in Kenya as well as other developing countries. The goal is to improve the livelihood and health status in vulnerable populations through nutrition, sanitation and hygiene programs. The 2013 Lancet series on maternal and child nutrition highlights key target areas to reduce chronic under nutrition by 20 million before $2020^{2,9-13}$. The progress towards achieving these targets can be tested through knowledge, attitudes and practice surveys. The rationale for this study was to understand local perceptions of the strengths and limitation of social development programs implemented in poor resource localities to improve public health outcomes. Here we hypothesized that community-based nutrition and health programs will improve public health nutrition, and so we sort to assess the impact of infant and young child nutrition community-based outreach programs in Suswa community.

\section{Materials and methods \\ Study Design}

The study was a cross-sectional survey. Data was collected in June 2013 using a mixed method approach. For quantitative data $(n=234)$ collection a structured questionnaire was used. For qualitative data collection, Focus Group Discussions (FGDs) were conducted using community members comprising (men and women of reproductive age as well as grandmothers to understand the local point of view. The rationale for including qualitative research was to enhance our understanding of the local community's perspectives, needs and context with regard to social programs.

\section{Study area and participants}

Narok County lies between latitudes $0^{\circ} 50^{\prime}$ South and longitude $35^{\circ} 28^{\prime}$ and $36^{\circ} 25^{\prime}$ East. The study site was Suswa location in Narok North Sub-County, Narok County, Kenya. Suswa location covers an area of approximately $500 \mathrm{~km}^{2}$ with a population estimate of 8,000 people in 25 villages, in which agro-pastoralism is the main economic activity. The target group included mothers and female caregivers with children aged 0-23 months (first 1,000 days of life) to capture information on knowledge, practices and health seeking behavior including maternal infant and young child nutrition (MIYCN), hygiene, and cultural beliefs that determines how a community utilizes health services.

\section{Sample size and sampling technique}

The sampling frame was drawn from Suswa location using a multistage and simple random sampling techniques. The sample size was calculated according to the infant and young child feeding (IYCF) indicators calculator as designed by CARE International in collaboration with others. This translated to 18 clusters of 13 households each giving a total of 234 households.In addition reserve clusters calculated at $5 \%$ non-response rate were sampled to replace any inaccessible cluster during the survey period. The various stages of sampling in Suswa location were sub-locations, villages and households. Clusters were defined as villages in Suswa location. In the second stage of sampling, households in each cluster were selected using the simple random method, using a list of all households with children below 23 months old. Using the table of random numbers thirteen households were selected in each cluster to attain the required sample size. Three focus groups of 8 men and women in the reproductive age as well as grandmothers were purposively selected from different clusters. Group 1 comprised of 4 women, 2 men and 2 grandmothers, group 2 had 5 women, 2 men and 1 grandmothers, while group 3 had 7 women, and 1 grandmothers to assure active participation ${ }^{15}$. Although a pretest was not conducted, the survey team was trained to ensure quality data is collected during the survey according to established protocol ${ }^{14}$. The survey team performed role-playing to familiarize themselves with the questionnaire. The main disadvantage of the study design was increased chance of error. To reduce the error, questionnaires and guides were translated and back translated to Maasai and English languages respectively.

\section{Data analysis}

For quantitative data, statistical inferences to larger populations' were calculated to allow the field staff to generalize findings to real situations and thus increase the external validity of the study findings in addition to descriptive statistics. The quantitative data were summarized by descriptive statistics and chi square test of independence to look for any associations between 
variables using Statistical Package for Social Sciences (SPSS) version 23.0. A p value of 0.05 was considered statistically significant. Qualitative content analysis was performed for qualitative data and the main themes were identified according to the common threads in the data.

\section{Ethical considerations}

Written and oral informed consent was obtained from all the participants prior to data collection. The Kenya Nutrition Information working Group, Institutional Review Board, approved the study protocol.

\section{Results}

Socio-demographic characteristics of study participants

A total of 234 households were included in the study at a 5\% non-response rate from 247 households. Socio-demographic characteristics of study participants are shown in Table 1. Majority of the participants were in the 21-30 years age bracket. Most mothers in the region have no formal education and the main source of income is pastoralism and subsistence farming. The main food crops grown in the area are maize and beans, which are consumed in an average household size of six people (data not shown).

Table 1. Demographic characteristics of the study participants

\begin{tabular}{lr}
\hline \hline Characteristic & \% (n= 234) \\
\hline Age (years) & \\
Below 20 & $19.9(47)$ \\
$21-30$ & $61.2(144)$ \\
Above 30 & $18.9(43)$ \\
\hline Education level & \\
No formal schooling & $62.1(146)$ \\
Primary school & $29.3(67)$ \\
Secondary & $6.0(15)$ \\
University & $2.6(6)$ \\
\hline Source of income & \\
Pastoralism and subsistence farming & $78.2(183)$ \\
Formal employment & $8.0(19)$ \\
Casual labor & $5.6(13)$ \\
Other sources (trade, hand-outs etc.) & $8.2(19)$ \\
\hline \hline
\end{tabular}

\section{Breastfeeding and malnutrition}

Since breast milk is known to protect newborn babies from infection due to maternally generated antibodies, we sort to determine the breastfeeding initiation time after birth. It was determined that although most mothers knew the importance of breastfeeding in the first hour immediately after birth (94.8\%), only about $44.4 \%$ of the infants were exclusively breastfed in the first six months (Table 2). However, there was no statistically significant difference $(p<0.05)$ between breastfeeding knowledge and level of education (Table 5). The reasons given from the FGDs for non-adherence to exclusive breastfeeding were subsequent pregnancies, unexplained refusal to suckle by the infant, childhood illnesses, insufficient breast milk, and fieldwork chores. 
Table 2. Level of knowledge and attitude as reported by study participants about breastfeeding, complementary feeding and childhood malnutrition

\begin{tabular}{lr}
\hline \hline Reason & \% (n) \\
\hline Knowledge and duration of breastfeeding & \\
Is initiation of breastfeeding at birth important? & $94.8(222 / 234)$ \\
Is exclusive breastfeeding (up to six months) important? & $\mathbf{4 4 . 4 ( 1 0 4 / 2 3 4 )}$ \\
Do you breastfeed your 6-23 months old baby & $79.3(186 / 234)$ \\
Do you breastfeed your 12-23 months old baby & $80.0(187 / 234)$ \\
\hline Knowledge about complementary feeding & \\
Right time to introduce complementary foods after 6-months & $60.9(143 / 234)$ \\
Introduction of complementary foods after 6-months & $67.8(159 / 234)$ \\
breastfeeding & \\
Feeding frequency of 3-4 to breastfed 6-23 months old & $80.0(187 / 234)$ \\
Feeding frequency of 3-4 to non-breastfed 6-23 months old & $79.0(185 / 234)$ \\
Special meals feeding during child illness & $73.0(171 / 234)$ \\
\hline Knowledge about malnutrition in children & \\
Is good nutrition beneficial to the mother during pregnancy? & $56.0(131 / 234)$ \\
Does good nutrition during lactation help children to grow? & $39.0(91 / 234)$ \\
Do you know the causes of malnutrition? & $58.0(136 / 234)$ \\
Do you know the signs of malnutrition? & $74.0(173 / 234)$ \\
Thinness & $\mathbf{5 9 . 0 ( 1 0 2 / 1 7 3 )}$ \\
Low weight & $\mathbf{4 2 . 0}(\mathbf{7 3 / 1 7 3 )}$ \\
Big belly & $9.0(16 / 173)$ \\
Brown hair & $19.0(33 / 173)$ \\
Lack of appetite & $33.0(57 / 173)$ \\
Is taking malnourished children to hospital beneficial? & $\mathbf{8 3 . 0 ( 1 9 4 / 2 3 4 )}$ \\
Should malnourished children be taken to hospital? & $\mathbf{8 2 . 0 ( 1 9 2 / 2 3 4 )}$ \\
\hline \hline bRespondents gave more than one reason for some characteristics
\end{tabular}

\section{Determinants of infant complementary feeding practices}

The study sort to assess whether complementary foods in addition to breast milk were introduced to the infant after six months of exclusive breastfeeding. Whereas $67.8 \%$ of the participants introduced complementary foods after six months of exclusive breastfeeding, only $60.9 \%$ had knowledge about the right time to introduce complementary foods, indicating that mothers practiced timely introduction of complementary foods compared to the level of awareness (Table 2). According to the FGD participants the main reason for early introduction of complementary foods was inadequate breast milk. The women participants also believe that when they are back in their homes in the evenings after work they do not have enough breast milk because of poor eating habits while away from home. The FGDs emphasized that it was the community's tradition to ensure children are breastfed for at least 2-years. A tradition that has been upheld in the community to date and greatly influences the feeding practice. The common foods given to children included porridge, fermented and fresh milk, mashed potatoes, rice, beans and fruits (based on season and availability).

\section{Knowledge on causes and signs of malnutrition}

Very little information is available on community-based nutrition programs dedicated to prevention of malnutrition and stunting in Suswa. Here, we focused on under-nutrition, the most common type of malnutrition that results from inappropriate maternal, infant and young child nutrition practices. About $74.1 \%$ of participants were aware of malnutrition signs, indicating that they are able to identify symptoms of malnutrition in children inasmuch as they had very limited knowledge with regards to malnutrition causes (Table 2). This is a great challenge to the community since some of the caregivers cannot address the causes of malnutrition in the household. About $82 \%$ of the participants indicated that they would take a malnourished child to hospital indicating that many participants were aware of the intervention strategies essential in the preven- 
tion and reduction of malnutrition. Regardless of this high indication there was a statistically significant difference $(p<0.05)$ between knowledge on causes and signs of malnutrition and level of education of study participants (Table 5). Suswa location had only one functional health facility; hence people resorted to herbal treatment. However, they visited the dispensary if the infections and disease persisted.

Knowledge and importance of vitamin A supplementation

Since vitamin A deficiency (VAD) compromises children's immune systems and the ability to fight common infections such as diarrhea and malaria, we sort to determine whether Suswa community members were aware that VAD is a cause of night blindness and a risk factor for infectious illnesses. The results indicate that $80.5 \%$ of the mothers had knowledge about vitamin A and VAD was a public health problem (Table 3). The vitamin A knowledgeable mothers were able to identify some of the health benefits as improved immunity and child growth $(32.6 \%)$ and night blindness prevention $(11.2 \%)$. Many of the participants $(90.9 \%)$ agreed that they took their children to the clinic for vitamin A supplementation. Given that vitamin supplementation has been shown to reduce child mortality, about $63.1 \%$ of the mothers had received post-partum Vitamin A supplementation within two weeks after delivery. In addition about $68.6 \%$ of children between $6-23$ months old had received a high-dose of oral Vitamin A supplementation as a preventive measure six months prior to the study.

Table 3. Maternal and nutrition healthcare practice management as reported by study participants

\begin{tabular}{|c|c|}
\hline Characteristic & $\%(n=234)$ \\
\hline \multicolumn{2}{|l|}{ Sources of nutrition information } \\
\hline Hospital health staff & $56.9(133)$ \\
\hline Community health worker & $4.2(10)$ \\
\hline Traditional birth attendant & $2.8(7)$ \\
\hline Women groups & $1.4(3)$ \\
\hline Mother support groups & $4.2(10)$ \\
\hline Mother in-law/ own mother & $23.6(55)$ \\
\hline $\mathrm{NGO} / \mathrm{CBO}$ & $4.2(10)$ \\
\hline Other sources & $2.7(6)$ \\
\hline \multicolumn{2}{|c|}{ Mid Upper Arm Circumference (MUAC) Measurement } \\
\hline Severe malnutrition $(<11.5)$ & $0.9(2 / 218)$ \\
\hline Moderate malnutrition (11.6-12.5) & $4.1(9 / 218)$ \\
\hline At risk of malnutrition (12.6- 13.5) & $9.6(21 / 218)$ \\
\hline Normal $(>13.6)$ & $85.3(186 / 218)$ \\
\hline \multicolumn{2}{|l|}{ Vitamin A Supplementation } \\
\hline $\begin{array}{l}\text { Take children for vitamin } \\
\text { supplementation }\end{array}$ & $90.0(211)$ \\
\hline Prevents night blindness & $12.0(28)$ \\
\hline Improves immunity & $33.0(77)$ \\
\hline Good for growth & $34.5(81)$ \\
\hline Good for eye sight & $1.5(4)$ \\
\hline No known benefit & $25.6(60)$ \\
\hline \multicolumn{2}{|l|}{ Number of antenatal clinic attendance } \\
\hline None & $4.4(10)$ \\
\hline Once & $2.3(5)$ \\
\hline Twice & $22.3(52)$ \\
\hline Thrice & $37.1(87)$ \\
\hline$>$ Three & $32.2(75)$ \\
\hline Don't know & $1.7(4)$ \\
\hline \multicolumn{2}{|l|}{ Mode of child delivery } \\
\hline Hospital nurse & $19.6(46)$ \\
\hline Traditional birth attendant & $28.6(67)$ \\
\hline Family/relatives & $50.9(119)$ \\
\hline Self & $0.9(2)$ \\
\hline
\end{tabular}




\section{Maternal health care utilization}

We also assessed maternal health care within the community and less than $60 \%$ of the households received nutrition and health information from hospital health workers. Other sources of nutrition information included mother in-laws, community health workers, traditional birth attendants (TBAs), women groups and Non Governmental Organizations (NGO). To monitor fetal growth and development and their own health, expectant mothers are expected to attend the antenatal clinics at least four times during pregnancy as recommended by the World Health Organization (WHO) clinical guidelines. Since most of the nutrition information is passed on during these antenatal clinics we determined the rate of attendance. Whereas $70 \%$ of the mothers attended the antenatal clinics, only $32.2 \%$ were within the WHO recommendation bracket of at least four antenatal care check-up (Table 3). Utilization of antenatal care services decreased significantly $(p<0.05)$ among those with low level of education (Table 5). A paltry $17 \%$ of the mothers had hospital delivery while the rest were home deliveries without professional health support services (Table 3). Home deliveries were attributed to expensive hospital bills as well as lack of transportation to the far off health dispensary. Surprisingly, we noted that the mothers had more confidence in the TBAs as well as other untrained people who performed home deliveries because of their long-term experience in conducting home deliveries. ThFGDs revealed that most of the mothers opted to deliver at home because the health facilities were very far and there were no means of transport, hence the need to walk for long distances to reach the facility. They also indicated that they had confidence in the people they chose to assist in delivery based on past experiences. The mothers also revealed that the community had trained TBAs who assisted in delivery. They also indicated that in certain situations the health workers were invited to conduct deliveries at home. The women through the FGD indicated that delivery in the hospital is very expensive as one required transportation to the hospital payment for the hospital services and thus not a good choice for most of them.

\section{Effect of sanitation and hygiene on nutritional out- comes}

Provision of adequate sanitation, good hygiene and proper food handling increases the likelihood that the child grows up healthy with little predisposition to enteric infections. About $70 \%$ of the community drinks dam water, while the remaining 30\% uses river, tap, borehole water, as well as water from protected and unprotected wells and water bowsers (Table 4). The FGDs participants reported that the water was neither treated at the source nor households (point of use). These water points are shared between humans and animals, thus increasing the possibility of zoonotic infections transmission from animals to humans. It was also noted that tap water is centrally placed at designated water kiosks in the community for quick access, although not sufficient to serve the whole community. There is a lack of waste disposal facilities in the community as $69.1 \%$ of the population defecates in the open (bush) and only $30.9 \%$ have access to pit latrines (Table 4). Because of the nomadic lifestyle, the Maasai dispose their waste in the bush and this has become part of their cultural practice as revealed by the FGDs. At least $85.3 \%$ of the study participants wash hands before food handling, $39.7 \%$ wash hands before feeding the child, 91\% use soap when washing hands and $52.6 \%$ wash hands after visiting the toilet (Table 4). However, the level of education had no significant $(\mathrm{p}=0.378)$ influence on hand washing after visiting the toilet (Table 5). 
Table 4: Water, sanitation and hygiene (WASH) practices as reported by study participants

\begin{tabular}{lr}
\hline \hline Characteristic & \% (n=234) \\
\hline Source of water & $\mathbf{6 9 . 8}(\mathbf{1 6 3})$ \\
Dam & $9.5(22)$ \\
Unprotected well & $4.3(10)$ \\
Protected well & $10.3(24)$ \\
River & $2.3(5)$ \\
Tap water & $2.3(5)$ \\
Water bowsers & $1.5(4)$ \\
Borehole & \\
\hline Hand washing practices & $52.6(123)$ \\
After visiting the toilet & $85.3(200)$ \\
Before handling food & $48.3(113)$ \\
After eating & $39.7(93)$ \\
Before feeding the child & $6.0(14)$ \\
During milking & \\
Hand washing with/without detergent & $91.0(213)$ \\
Soap and water & $9.0(21)$ \\
Water only & $70.0(164)$ \\
\hline Waste disposal and clinical outcomes & $30.0(70)$ \\
Open defecation & $7.7(18)$ \\
Use of pit latrines & $36.5(85)$ \\
Diarrhea in the week before study & $38.5(90)$ \\
Fever in the week before study & \\
Cough in the week before study & \\
\hline \hline
\end{tabular}

Table 5. Effect of education level on various nutritional outcomes as reported by study participants

\begin{tabular}{lrr}
\hline \hline \multicolumn{1}{c}{ Reason } & $\begin{array}{c}\text { Pearson } \\
\text { Chi- } \\
\text { square (X) }\end{array}$ & 'P<0.05 \\
\hline Exclusive breastfeeding knowledge & 6.562 & 0.584 \\
Introduction of complementary foods & 15.065 & 0.520 \\
Mid Upper Arm Circumference $\quad$ (MUAC) & 9.212 & 0.685 \\
measurement & & \\
Knowledge of malnutrition signs & $\mathbf{1 4 . 8 0 4}$ & $\mathbf{0 . 0 0 5}$ \\
Knowledge of malnutrition causes & $\mathbf{2 2 . 7 6 3}$ & $\mathbf{0 . 0 0 4}$ \\
Malnutrition actions taken & 26.592 & 0.147 \\
Health and nutrition information seeking behavior & 11.558 & 0.482 \\
Knowledge of Vitamin A supplementation & $\mathbf{1 7 . 5 4 4}$ & $\mathbf{0 . 0 2 5}$ \\
Importance of antenatal clinic attendance & $\mathbf{7 1 . 6 2 3}$ & $\mathbf{0 . 0 0 1}$ \\
Importance of hand washing after visiting the toilet & 4.215 & 0.378 \\
Type of waste disposal & $\mathbf{6 9 . 0 1 5}$ & $\mathbf{0 . 0 0 1}$ \\
\hline \hline
\end{tabular}

'Statistical testing by chi square for contingency presentations $(P<0.05$ values are significantly different) 


\section{Discussion}

Women with formal education are more likely to embrace beneficial feeding practices that would improve the health of their children compared to those without formal education. The low literacy levels in the study group greatly compromises the ability to make informed decisions with regards to infant and young child feeding practices. Even though a lot of nutrition information is disseminated to the community through NGOs, CBOs and Ministry of Health, assimilation is probably hampered by poor literacy. Exclusive breastfeeding was not possible because most mothers had to resume their household chores (fetching water and herding animals) soon or a month after delivery. Hence, the infants are left at home with grandmothers or older children who eventually feed them complementary foods including water, animal milk and butterfat obtained by churning milk. This is corroborated by other studies in poor resource countries where exclusive breastfeeding is particularly low ${ }^{16,17}$. There is need to emphasize the health benefits of exclusive breastfeeding to the Suswa community as exemplified by a study on the effects of breastfeeding promotion interventions in Pakistan ${ }^{11,18}$. The knowledge, which mothers possess, highly influences the practices associated with improved child nutrition. However, cultural practices increase the risk of malnutrition regardless of whether the mother is knowledgeable or not. For instance introduction of complementary foods was not dependent on the mother's knowledge about the practice, which is in agreement with a Vietnamese study ${ }^{19}$. Early complementary feeding among the Maasai is probably due to the household chores that the mothers undertake even when breastfeeding. This work burden creates the need for early complementary feeding, so that the children can be left in the care of other family members when the mothers go out to work. As a consequence exclusive breastfeeding rates drop down reducing the chances of improving child nutrition in the community ${ }^{3,6,20,21}$.

Infants aged 6-23 months are a very critical period and if inappropriate complementary foods, in terms of quality and quantity are provided, the infants are likely to be malnourished ${ }^{22,23}$. When compounded with poor health care practices, these can lead to infections that in turn aggravate malnutrition. Breastfeeding alone after six months is inadequate to ensure optimal growth of the child and if nutrient deprivation is prolonged the child is at risk of growth faltering ${ }^{3}$. In this study complementary feeding is well practiced, although the children have a high risk of developing malnutrition due to lack of food diversity. Data shows that malnutrition within the first 1000 days limits cognitive and physical development as well as educational achievement and economic productivity and should be continuously monitored ${ }^{24}$. The response to vitamin A supplementation is encouraging, and this is necessary because of increased vitamin A requirements during lactation. Unfortunately, vitamin A rich fruits and vegetables are not readily available and most people in the community cannot afford to buy the little that is available at expensive market prices increasing the risk of $\mathrm{VAD}^{25,26}$. Knowledge about the importance of vitamin A in the diet is currently minimal and can be attributed to inadequate vitamin A rich foods including liver, spinach, carrots, sweet potato and kales, a problem that requires more attention when such community nutrition programs are implemented ${ }^{27}$. According to the Maasai community customs, the male is the household head responsible for making family decisions with regard to resource allocation and financial expenditure. They make choices for their families based on financial status and inadequate nutritional knowledge limiting the women's ability to purchase nutrient-dense fruits and vegetables to supplement the meat and milk from livestock ${ }^{28}$. In the long run the women do not get to feed their children nutritious complementary foods. Women in these pastoral communities are marginalized and lack authority to make decisions with regard to resource allocation making it difficult to alleviate micronutrient deficiencies and stunting. Therefore to enhance adaptation of infant and child nutrition interventions, the men should also be involved in nutrition sensitization programs as a target group to reduce the gap of existing inequalities between men and women in such communities ${ }^{29}$.

Inasmuch as the TBAs perform home deliveries in the study community, they do not conduct home visits to promote newborn care. Because of this lack of supervision, mothers are quick to introduce complementary foods instead of practicing exclusive breastfeeding in the first six months of life. Low numbers of hospital deliveries indicate that implementation of baby friendly hospital initiative (BFHI), which supports exclusive breastfeeding practices may be difficult to scale up in the future in this region. Although the women seem to be knowledgeable, sanitation and hygiene practices for improved child nutrition are still below the WHO nutrition intervention targets ${ }^{1,30}$. Most of the community members are unaware of the dangers of unhygienic 
waste disposal, which enhances chances of exposure to enteric infections from water borne pathogens ${ }^{31}$. Most of the dam water is collected in the rainy season and is prone to contamination by waterborne microorganisms from fecal matter. Unfortunately the dam water used for domestic consumption is untreated, and this exposes the households to pathogens, predisposing the young children to diarrheal diseases. Many recent studies have shown that availability of health services as well as hygienic environments can reduce the risk and burden of childhood infections, including diarrhoea and malnutrition that are now associated with stunting ${ }^{31-33}$. Accessibility to clean water, sanitation and hygiene practices, environmental safety and availability of health care ensure that children are raised in healthy environments that do not preclude their growth and development. Children living in contaminated household environments due to open defecation are more prone to parasitic and bacterial infections and a high prevalence of environmental enteric dysfunction (EED) - a disorder featuring a small bowel with abnormal morphology and physiology ${ }^{31,34-37}$. Recent studies are now associating under-nutrition, marasmus and kwashiorkor with EED. This study findings are in agreement with a study by Onyango and coworkers that children in settings with poor sanitation and inadequate water supply be continuously breastfed for at least 2 years ${ }^{38}$.

\section{Conclusion}

Given the level of knowledge, attitude and public health practices among women in Narok, more sustainable and effective essential nutrition actions are needed. Lack of information, shortage of qualified health personnel, inadequate infrastructure and poor accessibility to health services hinders improved childhood nutrition. Gender equity can improve uptake of information, but new approaches such as empowering women to own livestock need to be addressed to influence maternal and child health. Community involvement in the design and implementation of culturally applicable education and nutrition programs to improve public health outcomes is crucial.

\section{Source of funding}

Concern Universal financially supported the study.

\section{Conflicts of interest}

The authors declare no conflict of interest.

\section{References}

1. Dewey KG, Cohen RJ, Rollins NC. WHO Technical
Background Paper: Feeding of nonbreastfed children from 6 to 24 months of age in developing countries. Food Nutr Bull. 2004;25(4):377-402.

2. Horton R, Lo S. Nutrition: A quintessential sustainable development goal. The Lancet. 2013. p. 371-2.

3. Haroon S, Das J, Salam R, Imdad A, Bhutta Z. Breastfeeding promotion interventions and breastfeeding practices: A systematic review. BMC Public Health [Internet]. 2013;13(Suppl 3):S20. Available from: http://www.biomedcentral.com/1471-2458/13/S3/ S20 \nhttp://www.pubmedcentral.nih.gov/articlerender.fcgi?artid $=3847366 \&$ tool $=$ pmcentre $\&$ \&render type $=$ abstract

4. Joshi N, Agho KE, Dibley MJ, Senarath U, Tiwari K. Determinants of inappropriate complementary feeding practices in young children in Nepal: secondary data analysis of Demographic and Health Survey 2006. Matern Child Nutr [Internet]. 2012;8:45-59. Available from: http://doi.wiley.com/10.1111/j.17408709.2011.00384.x

5. Kimani-Murage EW, Madise NJ, Fotso JC, Kyobutungi C, Mutua MK, Gitau TM, et al. Patterns and determinants of breastfeeding and complementary feeding practices in urban informal settlements, Nairobi Kenya. BMC Public Health [Internet]. 2011;11(1):396. Available from: http://www.ncbi.nlm.nih.gov/entrez/ query.fcgi? $\mathrm{cmd}=$ Retrieve $\& \mathrm{db}=$ PubMed\&dopt $=$ Citation\&list_uids $=21615957$

6. Agunbiade OM, Ogunleye O V. Constraints to exclusive breastfeeding practice among breastfeeding mothers in Southwest Nigeria: Implications for scaling up. Int Breastfeed J [nternet]. 2012 Apr 23;7(1):5. Available from: http://internationalbreastfeedingjournal.biomedcentral.com/articles/10.1186/1746-4358-7-5

7. Fabrizio CS, van Liere M, Pelto G. Identifying determinants of effective complementary feeding behaviour change interventions in developing countries. Matern Child Nutr. 2014;10(4):575-92.

8. Black RE, Alderman H, Bhutta ZA, Gillespie S, Haddad L, Horton S, et al. Maternal and child nutrition: Building momentum for impact. The Lancet. 2013. p. 372-5.

9. Black RE, Victora CG, Walker SP, Bhutta ZA, Christian P, De Onis M, et al. Maternal and child undernutrition and overweight in low-income and middle-income countries. Lancet. 2013;382(9890):427-51.

10. Bhutta ZA, Das JK, Rizvi A, Gaffey MF, Walker N, Horton S, et al. Evidence-based interventions for improvement of maternal and child nutrition: What can be done and at what cost? Lancet. 2013;382(9890):452-77. 11. Bhutta ZA, Ali S, Cousens S, Ali TM, Haider 
BA, Rizvi A, et al. Interventions to address maternal, newborn, and child survival: what difference can integrated primary health care strategies make? Lancet. 2008;372(9642):972-89.

12. Gillespie S, Haddad L, Mannar V, Menon P, Nisbett $\mathrm{N}$. The politics of reducing malnutrition: Building commitment and accelerating progress. Lancet. 2013;382(9891):552-69.

13. Ruel MT, Alderman H. Nutrition-sensitive interventions and programmes: How can they help to accelerate progress in improving maternal and child nutrition? Lancet. 2013;382(9891):536-51.

14. Ashworth A, Shrimpton R, Jamil K. Growth monitoring and promotion: review of evidence of impact. Matern Child Nutr [Internet]. 2008 Apr;4 Suppl 1(s1):86117. Available from: http://doi.wiley.com/10.1111/ j.1740-8709.2007.00125.x

15. Faber M, Kruger HS. Nutrition research in rural communities: application of ethical principles. Matern Child Nutr [nternet]. 2013;9(4):435-51. Available from: http://www.ncbi.nlm.nih.gov/pubmed/22591024

16. Lauer JA, Betrán AP, Victora CG, de Onís M, Barros AJ. Breastfeeding patterns and exposure to suboptimal breastfeeding among children in developing countries: review and analysis of nationally representative surveys. BMC Med [Internet]. 2004;2(1):26. Available from: http://bmcmedicine.biomedcentral.com/articles/10.1186/1741-7015-2-26

17. Ulak M, Chandyo RK, Mellander L, Shrestha PS, Strand TA. Infant feeding practices in Bhaktapur, Nepal: a cross-sectional, health facility based survey. Int Breastfeed J [Internet]. 2012;7(1):1. Available from: http:/ /internationalbreastfeedingjournal.biomedcentral.com/articles/10.1186/1746-4358-7-1

18. Imdad A, Yakoob MY, Bhutta ZA. Effect of breastfeeding promotion interventions on breastfeeding rates, with special focus on developing countries. $B M C$ Public Health [Internet]. 2011;11(Suppl 3):S24. Available from: http://bmcpublichealth.biomedcentral.com/articles/10.1186/1471-2458-11-S3-S24

19. Duong DV, Binns CW, Lee AH. Breast-feeding initiation and exclusive breast-feeding in rural Vietnam [Internet]. Public Health Nutrition. 2004. p. 795-9. Available from: http://ovidsp.ovid.com/ovidweb. $\operatorname{cgi} ? \mathrm{~T}=\mathrm{JS} \& \mathrm{PAGE}=$ reference $\& \mathrm{D}=$ emed $6 \& \mathrm{NEWS}=\mathrm{N}$ $\& A N=2004373515$

20. Onah S, Osuorah DI, Ebenebe J, Ezechukwu C, Ekwochi U, Ndukwu I. Infant feeding practices and maternal socio-demographic factors that influence practice of exclusive breastfeeding among mothers in
Nnewi South-East Nigeria: a cross-sectional and analytical study. Int Breastfeed J [Internet]. 2014;9(1):6. Available from: http://internationalbreastfeedingjournal.biomedcentral.com/articles/10.1186/1746-4358-9-6

21. Bhandari N, Kabir IAKM, Salam MA. Mainstreaming nutrition into maternal and child health programmes: Scaling up of exclusive breastfeeding. Maternal and Child Nutrition. 2008. p. 5-23.

22. Waswa LM, Jordan I, Herrmann J, Krawinkel MB, Keding GB. Community-based educational intervention improved the diversity of complementary diets in western Kenya: results from a randomized controlled trial. Public Health Nutr [Internet]. 2015;18(18):3406-19. Available from: http://www.journals.cambridge.org/ abstract_S1368980015000920

23. Daelmans B, Ferguson E, Lutter CK, Singh N, Pachón $\mathrm{H}$, Creed-Kanashiro $\mathrm{H}$, et al. Designing appropriate complementary feeding recommendations: Tools for programmatic action. Matern Child Nutr. 2013;9(S2):116-30.

24. Hoddinott J, Alderman H, Behrman JR, Haddad $\mathrm{L}$, Horton S. The economic rationale for investing in stunting reduction. Matern Child Nutr. 2013;9(S2):69-82. 25. Filteau SM, Tomkins AM. Promoting vitamin A status in low-income countries. Lancet. 1999. p. 1458-9. 26. Haider BA, Bhutta ZA. Neonatal vitamin A supplementation for the prevention of mortality and morbidity in term neonates in developing countries. Cochrane Database Syst Rev [Internet]. 2011;CD006980. Available from: http://www.ncbi.nlm.nih.gov/pubmed/21975758

27. Kuchenbecker J, Reinbott A, Mtimuni B, Krawinkel MB, Jordan I. Nutrition education improves dietary diversity of children 6-23 months at community-level: Results from a cluster randomized controlled trial in Malawi. PLoS One. 2017;12(4).

28. Mukuria AG, Martin SL, Egondi T, Bingham A, Thuita FM. Role of Social Support in Improving Infant Feeding Practices in Western Kenya: A Quasi-Experimental Study. Glob Heal Sci Pract. 2016;4(1):55-72.

29. Muehlhoff E, Wijesinha-Bettoni R, Westaway E, Jeremias T, Nordin S, Garz J. Linking agriculture and nutrition education to improve infant and young child feeding: Lessons for future programmes. Matern Child Nutr [Internet]. 2017;13(April 2016):e12411. Available from: http://doi.wiley.com/10.1111/mcn.12411

30. WHO. Indicators for assessing infant and young child feeding practices [Internet]. Part 1-definitions: conclusions of a consensus meeting held 6-8 November 2007. 2008. Available from: http://www.who.int/maternal_child_adolescent/documents/9789241596664/ en/ 
31. Mbuya MNN, Humphrey JH. Preventing environmental enteric dysfunction through improved water, sanitation and hygiene: An opportunity for stunting reduction in developing countries. Maternal and Child Nutrition. 2016. p. 106-20.

32. Ngure FM, Reid BM, Humphrey JH, Mbuya MN, Pelto G, Stoltzfus RJ. Water, sanitation, and hygiene (WASH), environmental enteropathy, nutrition, and early child development: Making the links. Ann N Y Acad Sci. 2014;1308(1):118-28.

33. Torlesse H, Cronin AA, Sebayang SK, Nandy R. Determinants of stunting in Indonesian children: evidence from a cross-sectional survey indicate a prominent role for the water, sanitation and hygiene sector in stunting reduction. BMC Public Health [Internet]. 2016;16(1):669. Available from: http://bmcpublichealth.biomedcentral. com/articles/10.1186/s12889-016-3339-8

34. Keusch GT, Rosenberg IH, Denno DM, Duggan C, Guerrant RL, Lavery J V., et al. Implications of acquired environmental enteric dysfunction for growth and stunting in infants and children living in low-and middle-income countries. Food Nutr Bull. 2013;34(3):357-64.

35. Mahfuz M, Das S, Mazumder RN, Masudur Rahman M, Haque R, Bhuiyan MMR, et al. Bangladesh Environmental Enteric Dysfunction (BEED) study: protocol for a community-based intervention study to validate non-invasive biomarkers of environmental enteric dysfunction. BMJ Open [Internet]. 2017;7(8):e017768. Available from: http://bmjopen.bmj.com/lookup/ doi/10.1136/bmjopen-2017-017768

36. Keusch GT, Denno DM, Black RE, Duggan C, Guerrant RL, Lavery J V., et al. Environmental enteric dysfunction: Pathogenesis, diagnosis, and clinical consequences. Clin Infect Dis. 2014;59:S207-12.

37. Crane RJ, Jones KDJ, Berkley JA. Environmental enteric dysfunction: An overview. Food Nutr Bull. 2015;36:S76-87.

38. Onyango AW, Esrey SA, Kramer MS. Continued breastfeeding and child growth in the second year of life: A prospective cohort study in western Kenya. Lancet. 1999;354(9195):2041-5. 